\title{
ON THE TRANSFORMATION OF DIFFUSION PROCESSES INTO THE WIENER PROCESS*
}

\author{
GEORGE W. BLUMAN†
}

\begin{abstract}
Necessary and sufficient conditions are given for transforming (constructively) a one-dimensional diffusion process described by a Kolmogorov equation into the Wiener process. These conditions are shown to be equivalent to invariance of a parabolic partial differential equation under a six-parameter Lie group of point transformations. Moreover these conditions correspond to a significant generalization of Cherkasov's result; i.e., a much wider class of Kolmogorov equations can be derived from the Wiener process than was previously realized.
\end{abstract}

1. Introduction. In this paper we find the most general one-dimensional diffusion process, whose transition probability density function (transition pdf) satisfies a Kolmogorov equation, which can be derived from the Wiener process. The one-to-one transformation relating the processes is given explicitly, leading to the construction of the corresponding transition pdf.

In his celebrated work, [4], Kolmogorov posed the problem we discuss. Cherkasov [3] gave a partial solution to Kolmogorov's question but he restricted himself to a special class of point transformations used by Kolmogorov in [4]. A recent paper, [6], interpreting Cherkasov's result, unfortunately has given the impression that Cherkasov found "necessary and sufficient conditions for transforming into the Wiener process a one-dimensional diffusion process described by a Kolmogorov equation".

By considering general point transformations we find necessary and sufficient conditions for relating the Wiener process to a one-dimensional diffusion process describing a transition pdf. In $\S 4$ Cherkasov's restriction on the coefficients is demonstrated to be a rather special case of our general result.

The Kolmogorov equation is a parabolic partial differential equation (p.d.e.). In the course of this paper we consider the invariance of parabolic p.d.e.'s under Lie groups of point transformations. It is shown that a one-dimensional linear parabolic p.d.e. is invariant under at most a six-parameter Lie group of point transformations. Moreover, as shown by Lie [5], a linear parabolic p.d.e. can be mapped, one-to-one, into the heat equation (the governing p.d.e. of a Wiener process) if and only if it is invariant under a six-parameter continuous group (Lie group) of point transformations. However Lie's proof corresponded to a classification scheme for parabolic p.d.e.'s. Explicitly, Lie neither determined the class of parabolic p.d.e.'s equivalent to the heat equation nor constructed the corresponding mapping. Here we give a direct proof which explicitly determines the class of such p.d.e.'s and constructs the mapping. Moreover our method of proof could be extended to other mapping problems.

As a consequence a diffusion process can be transformed into a Wiener process if and only if the corresponding Kolmogorov equation is invariant under a six-parameter Lie group of point transformations. In applying our results to a specific Kolmogorov equation it may be easier to first check whether its coefficients lead to six-parameter invariance rather than to check whether its coefficients satisfy the equations developed in $\S 4$.

* Received by the editors September 4, 1979, and in final form November 23, 1979.

$\dagger$ Department of Mathematics and Institute of Applied Mathematics and Statistics, University of British Columbia, Vancouver, B.C. V6T 1W5, Canada. 
2. Some preliminaries. Let $f(t, x ; u, y)$ be the transition pdf of a Markov process satisfying a Kolmogorov equation

$$
\frac{\partial f}{\partial t}+a(t, x) \frac{\partial^{2} f}{\partial x^{2}}+b(t, x) \frac{\partial f}{\partial x}=0,
$$

for $x$ in some interval $I, t<u$, with initial condition

$$
\lim _{t \rightarrow u} f(t, x ; u, y)=\delta(x-y)
$$

where $\delta$ is the Dirac delta function.

Our aim is to find all possible types of the coefficients $\{a(t, x), b(t, x)\}$ so that $\{(2.1),(2.2)\}$ can be transformed, in a one-to-one manner, to a Wiener process whose transition pdf $\bar{f}(\bar{t}, \bar{x} ; \bar{u}, \bar{y})$ satisfies the "backward" heat equation

$$
\frac{\partial \bar{f}}{\partial \bar{t}}+\frac{\partial^{2} \bar{f}}{\partial \bar{x}^{2}}=0
$$

for $\bar{x}$ in some corresponding interval $\bar{I}, \bar{t}<\bar{u}$, with initial condition

$$
\lim _{\bar{t} \rightarrow \bar{u}} \bar{f}(\bar{t}, \bar{x} ; \bar{u}, \bar{y})=\delta(\bar{x}-\bar{y}) .
$$

Cherkasov [3] solved a limited form of Kolmogorov's problem where he restricted himself to one-to-one point transformations of the form

$$
\bar{x}=\psi(t, x), \quad \bar{t}=\zeta(t), \quad \bar{f}=f \cdot\left[\frac{\partial \psi(u, y)}{\partial y}\right]^{-1} .
$$

In this paper we seek the most general one-to-one point transformation of the form

$$
\bar{t}=T(t, x, f), \quad \bar{x}=X(t, x, f), \quad \bar{f}=F(t, x, f),
$$

and corresponding coefficients $\{a(t, x), b(t, x)\}$, so that $\{(2.1),(2.2)\}$ is equivalent to $\{(2.3),(2.4)\}$. In seeking such a mapping formula, $(2.6),\{y, u\}$ are related to $\{\bar{y}, \bar{u}\}$ according to it.

If we let

$$
x^{\prime}=\phi(t, x), \quad t^{\prime}=t
$$

where

$$
\phi(t, x)=\int_{x_{0}}^{x}[a(t, z)]^{-1 / 2} d z,
$$

for some arbitrary $x_{0} \in I$, then (2.1) becomes

$$
\frac{\partial f}{\partial t^{\prime}}+\frac{\partial^{2} f}{\partial\left(x^{\prime}\right)^{2}}+D\left(t^{\prime}, x^{\prime}\right) \frac{\partial f}{\partial x^{\prime}}=0,
$$

where

$$
D\left(t^{\prime}, x^{\prime}\right)=\frac{\partial \phi}{\partial t}+a(t, x) \frac{\partial^{2} \phi}{\partial x^{2}}+b(t, x) \frac{\partial \phi}{\partial x} .
$$

If we let $\left\{C\left(t^{\prime}, x^{\prime}\right), f^{\prime}\right\}$ be such that

$$
\frac{\partial C}{\partial x^{\prime}}=-\frac{1}{2} D\left(t^{\prime}, x^{\prime}\right)
$$


and

$$
f^{\prime}=e^{-C} f
$$

we find that $(2.1)$ is equivalent to

$$
\frac{\partial f^{\prime}}{\partial t^{\prime}}+\frac{\partial^{2} f^{\prime}}{\partial\left(x^{\prime}\right)^{2}}+G\left(t^{\prime}, x^{\prime}\right) f^{\prime}=0
$$

where

$$
G\left(t^{\prime}, x^{\prime}\right)=\frac{\partial C}{\partial t^{\prime}}+\frac{\partial^{2} C}{\partial\left(x^{\prime}\right)^{2}}-\left(\frac{\partial C}{\partial x^{\prime}}\right)^{2} .
$$

Our problem now reduces to finding the most general $G\left(t^{\prime}, x^{\prime}\right)$ and mapping (2.6), so that $(2.13)$ with

$$
\lim _{t^{\prime} \rightarrow u^{\prime}} f^{\prime}\left(t^{\prime}, x^{\prime} ; u^{\prime}, y^{\prime}\right)=\left[e^{-C\left(u^{\prime}, y^{\prime}\right)} \frac{\partial \phi}{\partial y}(u, y)\right] \delta\left(x^{\prime}-y^{\prime}\right)
$$

can be transformed one-to-one into $\{(2.3),(2.4)\}$, where $y^{\prime}=\phi(u, y), u^{\prime}=u$.

3. Construction of the mapping. In this section, for convenience of notation, we unprime the primed variables of $\S 2$. First we prove a theorem concerning a group theoretical property of p.d.e.'s of the form (2.13).

THEOREM 1. (2.13) is invariant under a six-parameter Lie group of point transformations if and only if $G(t, x)$ is of the form

$$
G(t, x)=q_{0}(t)+q_{1}(t) x+q_{2}(t) x^{2}
$$

where $\left\{q_{0}(t), q_{1}(t), q_{2}(t)\right\}$ are arbitrary functions of $t$.

Proof. Say the one-parameter $(\varepsilon)$ Lie group of point transformations,

$$
\begin{aligned}
& t^{*}=t+\varepsilon \tau(t, x, f)+O\left(\varepsilon^{2}\right), \\
& x^{*}=x+\varepsilon \xi(t, x, f)+O\left(\varepsilon^{2}\right), \\
& f^{*}=f+\varepsilon \pi(t, x, f)+O\left(\varepsilon^{2}\right),
\end{aligned}
$$

leaves invariant (2.13). Then the determining equations [1] for the group infinitesimals $\{\tau, \xi, \pi\}$ reduce to

$$
\tau=\tau(t), \quad \xi=\xi(t, x), \quad \pi=g(t, x) f,
$$

where (a prime corresponds to differentiation with respect to $t$ ),

$$
\begin{aligned}
& \xi(t, x)=\frac{1}{2} x \tau^{\prime}(t)+A(t), \\
& g(t, x)=\frac{x^{2} \tau^{\prime \prime}(t)}{8}+\frac{1}{2} x A^{\prime}(t)+B(t),
\end{aligned}
$$

and, with respect to a given $G(t, x),\{\tau(t), A(t), B(t)\}$ must satisfy the differential equation

$$
\begin{aligned}
& \frac{x^{2}}{8} \tau^{\prime \prime \prime}(t)+\frac{1}{4} \tau^{\prime \prime}(t)+\frac{1}{2} x A^{\prime \prime}(t)+B^{\prime}(t) \\
& \quad+\left[\frac{1}{2} x \tau^{\prime}(t)+A(t)\right] \frac{\partial G}{\partial x}+\tau(t) \frac{\partial G}{\partial t}+\tau^{\prime}(t) G=0 .
\end{aligned}
$$


From (3.5) we see that at most a six-parameter Lie group of point transformations leaves invariant (2.13) since, respectively, there are at most three arbitrary constants for $\tau(t)$, at most two for $A(t)$, and at most one for $B(t)$ in the solution of (3.5) for a given $G(t, x)$. Moreover it is clear that $G(t, x)$ must be analytic in $x$ for six-parameter invariance. After representing $G(t, x)$ in a power series in $x$, from (3.5) it is easy to show that (2.13) is invariant under a six-parameter Lie group of point transformations if and only if

$$
\frac{\partial^{3} G}{\partial x^{3}}=0
$$

i.e., $G(t, x)$ satisfies (3.1). This completes the proof of Theorem 1 .

If $G(t, x)$ satisfies (3.1), the resulting equations satisfied by $\{\tau(t), A(t), B(t)\}$ are:

$$
\begin{aligned}
& \frac{\tau^{\prime \prime \prime}}{8}+2 q_{2}(t) \tau^{\prime}+q_{2}^{\prime}(t) \tau=0, \\
& \frac{1}{2} A^{\prime \prime}+2 q_{2}(t) A=-\left(\frac{3}{2} q_{1}(t) \tau^{\prime}+q_{1}^{\prime}(t) \tau\right), \\
& B^{\prime}=-\left[\frac{1}{4} \tau^{\prime \prime}+q_{0}^{\prime}(t) \tau+q_{0}(t) \tau^{\prime}+q_{1}(t) A\right] .
\end{aligned}
$$

Next we prove a lemma concerning a necessary condition for the existence of a mapping between a Kolmogorov equation (2.1) and the backward heat equation (2.3).

LEMMA. If it is possible to map (2.1) or, equivalently (2.13), into (2.3) by a one-to-one point transformation, then $G(t, x)$ must be of the form (3.1); i.e., (2.1) must be invariant under a six-parameter Lie group of point transformations.

Proof. The backward heat equation (2.3) is invariant under the six-parameter $\left\{\alpha_{1}, \alpha_{2}, \cdots, \alpha_{6}\right\}$ Lie group of point transformations [1], [2]:

$$
\begin{aligned}
& (\bar{t})^{*}=\bar{t}+\varepsilon \bar{\tau}(\bar{t})+O\left(\varepsilon^{2}\right), \\
& (\bar{x})^{*}=\bar{x}+\varepsilon \bar{\xi}(\bar{t}, \bar{x})+O\left(\varepsilon^{2}\right), \\
& (\bar{f})^{*}=\bar{f}+\varepsilon \bar{g}(\bar{t}, \bar{x}) \bar{f}+O\left(\varepsilon^{2}\right),
\end{aligned}
$$

where

$$
\begin{aligned}
& \bar{\tau}(\bar{t})=-\alpha_{1}+2 \alpha_{2} \bar{t}-\alpha_{3}(\bar{t})^{2}, \\
& \bar{\xi}(\bar{t}, \bar{x})=\alpha_{2} \bar{x}-\alpha_{3} \bar{x} \bar{t}+\alpha_{4}-\alpha_{5} \bar{t}, \\
& \bar{g}(\bar{t}, \bar{x})=\alpha_{3}\left[\frac{1}{2} \bar{t}-\frac{1}{4}(\bar{x})^{2}\right]-\frac{1}{2} \alpha_{5} \bar{x}+\alpha_{6} .
\end{aligned}
$$

A one-to-one point transformation of the form (2.6) relating solutions of (2.13) to those of (2.3) must in turn induce a one-to-one mapping which relates the Lie group of point transformations of $(2.13)$ to that of $(2.3)$ by some appropriate representation of their respective parameters. Hence the existence of such a mapping requires that point Lie groups of (2.13) and (2.3) have the same number of parameters, completing the proof of the lemma.

We are now ready to solve the problem posed at the end of $\S 2$. In particular, in the following theorem we show that the necessary condition of our lemma is also a sufficient condition, so that a Kolmogorov equation can be mapped one-to-one into the backward heat equation if and only if it is invariant under a six-parameter Lie group of point transformations. Moreover we explicitly construct the mapping and show that we never have to solve $\{(3.6),(3.7),(3.8)\}$ in order to construct the mapping.

THEOREM 2. If $G(t, x)$ is of the form (3.1) then we can transform (2.13) to (2.3), constructively, by a one-to-one mapping. 
Proof. We prove this theorem by showing how to construct a one-to-one mapping relating solutions of (2.13) to those of (2.3). The procedure we follow is first to demand that a mapping (2.6) transform the group of (2.13) into the group of (2.3). This idea is used to construct such a mapping. One can then demonstrate that this mapping does indeed transform (2.13) into (2.3) in a one-to-one manner. We now proceed.

Clearly we must show how to construct the required mapping without explicitly computing the solution of $\{(3.6),(3.7),(3.8)\}$, i.e., $\{\tau(t), A(t), B(t)\}$. Let

$$
\tau=\sum_{i=1}^{3} \alpha_{i} \tau_{i}(t)
$$

represent the general solution of (3.6) where $\left\{\tau_{1}(t), \tau_{2}(t), \tau_{3}(t)\right\}$ are linearly independent solutions of (3.6) and $\left\{\alpha_{1}, \alpha_{2}, \alpha_{3}\right\}$ are arbitrary constants.

Assuming the form (3.11) for $\tau(t)$, we let

$$
A=\sum_{i=1}^{5} \alpha_{i} A_{i}(t)
$$

represent the general solution of (3.7), where

$$
\begin{aligned}
& \frac{1}{2} A_{i}^{\prime \prime}+2 q_{2}(t) A_{i}=0, \quad i=4,5, \\
& \frac{1}{2} A_{i}^{\prime \prime}+2 q_{2}(t) A_{i}=-\left[\frac{3}{2} q_{1}(t) \tau_{i}^{\prime}+q_{1}^{\prime}(t) \tau_{i}\right], \quad i=1,2,3,
\end{aligned}
$$

and $\left\{\alpha_{4}, \alpha_{5}\right\}$ are additional arbitrary constants. Here $\left\{A_{4}(t), A_{5}(t)\right\}$ are linearly independent solutions of the homogeneous part of (3.7); $A_{i}(t)$ is a particular solution of (3.7) corresponding to the input $\tau_{i}(t)$ for $i=1,2,3$.

Assuming (3.11) and (3.12abc), we let

$$
B=\sum_{i=1}^{6} \alpha_{i} B_{i}(t)
$$

represent the general solution of (3.8), where

(3.13b) $\quad B_{6}^{\prime}=0$,

(3.13c) $\quad B_{i}^{\prime}=-q_{1}(t) A_{i}, \quad i=4,5$,

$$
B_{i}^{\prime}=-\left[q_{1}(t) A_{i}+\frac{1}{4} \tau_{i}^{\prime \prime}+q_{0}^{\prime}(t) \tau_{i}+q_{0}(t) \tau_{i}^{\prime}\right], \quad i=1,2,3,
$$

and $\alpha_{6}$ is an additional arbitrary constant. Here $B_{6}(t)$ is a solution of the homogeneous part of $(3.8) ; B_{i}(t)$ is a particular solution of (3.8), corresponding to the input $A_{i}(t)$ for $i=4,5$; and to the input $\tau_{i}(t)$ with corresponding $A_{i}(t)$ for $i=1,2,3$.

Each of the parameters $\left\{\alpha_{1}, \alpha_{2}, \cdots, \alpha_{6}\right\}$ corresponds to a one-parameter subgroup of the six-parameter Lie group of point transformations.

It turns out that the mapping (2.6) reduces to the form

$$
\text { (3.14a, b, c) } \quad \bar{t}=T(t), \quad \bar{x}=X(t, x), \quad \bar{f}=F(t, x) f \text {. }
$$

If (3.14abc) maps (2.13) into (2.3) then, by some appropriate representation, it is necessary that (3.14) maps, parameter by parameter, the group of (2.13) into the group of (2.3). Hence after transforming $(3.14 \mathrm{abc})$ by the group of $(2.13)$ the resulting infinitesimals must be those of (2.3). This leads to differential equations satisfied by $\{T(t), X(t, x), F(t, x)\}$ whose solutions are shown to lead to our mapping. The parameter representation we use is the direct one of associating respectively the parameters $\left\{\alpha_{1}, \alpha_{2}, \cdots, \alpha_{6}\right\}$ with those used to describe the group of the backward heat equation (3.10). 
After respectively equating the coefficients of each of the parameters $\left\{\alpha_{1}, \alpha_{2}, \alpha_{3}\right\}$ from the $O(\varepsilon)$ terms in the expansion of $(\bar{t})^{*}=T\left(t^{*}\right)$, we find that $\left\{\tau_{1}(t), \tau_{2}(t), \tau_{3}(t), T(t)\right\}$ satisfy

$$
d T=\frac{-d t}{\tau_{1}}, \quad \frac{d T}{2 T}=\frac{d t}{\tau_{2}}, \quad \frac{d T}{T^{2}}=\frac{-d t}{\tau_{3}} .
$$

The consistency relations corresponding to (3.6) and (3.15) lead to $T(t)$ satisfying the grand equation

$$
2 T^{\prime \prime \prime}\left(T^{\prime}\right)^{3}-3\left(T^{\prime}\right)^{2}\left(T^{\prime \prime}\right)^{2}-16 q_{2}(t)\left(T^{\prime}\right)^{4}=0
$$

Note that if $M=T^{\prime \prime} / T^{\prime}=d / d t\left(\log \left|T^{\prime}\right|\right)$, then (3.16) reduces to a Ricatti equation for $M(t)$, namely,

$$
2 M^{\prime}=(M)^{2}+16 q_{2}(t) .
$$

Hence if $q_{2}(t) \geqq 0$ for all $t$, then $T^{\prime}(t)$ is a monotone function, and thus a solution $T(t)$ of (3.16) defines a one-to-one mapping.

After respectively equating the coefficients of $\left\{\alpha_{4}, \alpha_{5}, \alpha_{2}, \alpha_{3}, \alpha_{1}\right\}$ from the $O(\varepsilon)$ terms in the expansion of $(\bar{x})^{*}=X\left(t^{*}, x^{*}\right)$, we find that $X(t, x)$ must satisfy the equations

$$
\begin{gathered}
A_{4} \frac{\partial X}{\partial x}=1, \\
A_{5} \frac{\partial X}{\partial x}=-T, \\
X-\left[\frac{1}{2} x \tau_{2}^{\prime}+A_{2}\right] \frac{\partial X}{\partial x}-\tau_{2} \frac{\partial X}{\partial t}=0, \\
T X+\left[\frac{1}{2} x \tau_{3}^{\prime}+A_{3}\right] \frac{\partial X}{\partial x}+\tau_{3} \frac{\partial X}{\partial t}=0, \\
{\left[\frac{1}{2} x \tau_{1}^{\prime}+A_{1}\right] \frac{\partial X}{\partial x}+\tau_{1} \frac{\partial X}{\partial t}=0 .}
\end{gathered}
$$

The solution of $\{(3.18),(3.19),(3.20)\}$ leads to

$$
X(t, x)=C_{0} x \sqrt{T^{\prime}(t)}+Y(t)
$$

where $Y(t)$ satisfies

$$
2 \frac{T}{T^{\prime}} Y^{\prime}-Y=-C_{0} \sqrt{T^{\prime}} A_{2}
$$

and $C_{0}$ is an arbitrary constant.

After equating the coefficients of $\alpha_{4}$ and $\alpha_{5}$ from the $O(\varepsilon)$ terms in the expansion of $(\bar{f})^{*}=F\left(t^{*}, x^{*}\right) f^{*}$, we find that $F(t, x)$ satisfies the equations

$$
\begin{aligned}
& A_{4} \frac{\partial F}{\partial x}+\left[\frac{1}{2} x A_{4}^{\prime}+B_{4}\right] F=0, \\
& A_{5} \frac{\partial F}{\partial x}+\left[\frac{1}{2} X+\frac{1}{2} x A_{5}^{\prime}+B_{5}\right] F=0 .
\end{aligned}
$$


This leads us to see that $C_{0}=1$ and that

$$
\begin{aligned}
& X(t, x)=x \sqrt{T^{\prime}}-2 B_{4} T-2 B_{5}, \\
& F(t, x)=W(t) \cdot \exp \left[\frac{x^{2}}{8} \frac{T^{\prime \prime}}{T^{\prime}}-x B_{4} \sqrt{T^{\prime}}\right],
\end{aligned}
$$

where

$$
\begin{aligned}
& B_{5}=\int_{t_{0}}^{t} q_{1}(s) T(s)\left[T^{\prime}(s)\right]^{-1 / 2} d s \\
& B_{4}=-\int_{t_{0}}^{t} q_{1}(s)\left[T^{\prime}(s)\right]^{-1 / 2} d s
\end{aligned}
$$

and $W(t)$ is arbitrary.

The coefficient of the $\alpha_{2}$ parameter in the $O(\varepsilon)$ terms in the expansion of $(\bar{f})^{*}=F\left(t^{*}, x^{*}\right) f^{*}$ results in $W(t)$ satisfying

$$
\tau_{2} W^{\prime}+\left[B_{2}-A_{2} B_{4} \sqrt{T^{\prime}}\right] W=0
$$

where

$$
\begin{gathered}
A_{2}=2 B_{4} T\left(T^{\prime}\right)^{-1 / 2}-2 B_{5}\left(T^{\prime}\right)^{-1 / 2}, \\
\tau_{2}=2 T\left(T^{\prime}\right)^{-1} .
\end{gathered}
$$

$B_{2}(t)$ satisfies $(3.13 \mathrm{~d})$ for $i=2$,

$$
B_{2}=-\frac{1}{4} \tau_{2}^{\prime}-2 B_{4} B_{5}-q_{0}(t) \tau_{2}+\lambda
$$

where $\lambda$ is an arbitrary constant, so that

$$
W(t)=\exp \int_{t_{0}}^{t}\left[\frac{A_{2}(s) B_{4}(s) \sqrt{T^{\prime}(s)}-B_{2}(s)}{\tau_{2}(s)}\right] d s .
$$

Since the constructed $F(t, x)>0$ for all $t, x$, and $\partial X / \partial x=\sqrt{T^{\prime}}$, we see that the constructed mapping is one-to-one where we use a solution to the grand equation (3.16) where $T^{\prime}(t)>0$ for all $t$. By direct calculation it is now easy to check that if $\lambda=\frac{1}{2}$ our constructed mapping is indeed a one-to-one mapping between (2.13) and (2.3), completing the proof of Theorem 2 .

We now close this section with a few remarks.

Remark 1. If $q_{2}(t) \equiv 0$, then it is easy to show that $T=\left(a_{1}+a_{2} t\right) /\left(t+a_{3}\right)$ where $\left\{a_{1}, a_{2}, a_{3}\right\}$ are arbitrary constants.

Remark 2. There is still a further degree of freedom in our mapping, namely that $C^{*} W(t)$ solves (3.30) for an arbitrary constant $C^{*}$ so that $\bar{f}=C^{*} F(t, x) f$. To map the diffusion process $\{(2.1),(2.2)\}$ into the Wiener process $\{(2.3),(2.4)\}$ requires that the source conditions map into each other. In terms of the variables of $\S 2$, this leads to

$$
C^{*}=e^{C\left(u^{\prime}, y^{\prime}\right)} \cdot\left[\frac{\partial \phi}{\partial y}(u, y)\right]^{-1}\left[T^{\prime}\left(u^{\prime}\right)\right]^{-1 / 2}\left[F\left(u^{\prime}, y^{\prime}\right)\right]^{-1} \text {. }
$$

Remark 3. Since every linear parabolic p.d.e. in one dimension can be mapped into the form (2.13) it follows that such a p.d.e. is equivalent to the heat equation if and only if it is invariant under a six-parameter Lie group of point transformations. This appears to be the easiest way to apply Theorem 2 to a given parabolic p.d.e. and in particular to Kolmogorov's equation (2.1); i.e., instead of computing $G(t, x)$ for a given 
parabolic p.d.e. it is better to first check whether the given p.d.e. is invariant under a six-parameter Lie group of point transformations. If this is the case then one should compute $G(t, x)$ and apply the mapping formulas.

Remark 4. There is a unique structure for the maximal six-parameter Lie group of point transformations which can leave invariant a one-dimensional linear parabolic partial differential equation, a result previously proved by Lie in his symmetry classification scheme for linear p.d.e.'s [5].

4. Connection with Cherkasov's result. Restricting himself to transformations of the form (2.4), and setting

$$
\begin{aligned}
& \alpha(t, x)=[a(t, x)]^{1 / 2}, \\
& \beta(t, x)=[a(t, x)]^{1 / 2} \int_{x_{0}}^{x}[a(t, z)]^{-1 / 2} d z, \\
& \gamma(t, x)=2 b(t, x)-\frac{\partial a(t, x)}{\partial x}-[a(t, x)]^{1 / 2} \int_{x_{0}}^{x} \frac{\partial a(t, z)}{\partial t}[a(t, z)]^{-3 / 2} d z,
\end{aligned}
$$

Cherkasov [3] showed that $\{(2.1),(2.2)\}$ maps one-to-one into $\{(2.3),(2.4)\}$ if and only if $\{\alpha(t, x), \beta(t, x), \gamma(t, x)\}$ satisfy the determinant equation

$$
\left|\begin{array}{ccc}
\alpha & \beta & \gamma \\
\alpha_{x} & \beta_{x} & \gamma_{x} \\
\alpha_{x x} & \beta_{x x} & \gamma_{x x}
\end{array}\right|=0,
$$

where $\alpha_{x}=\partial \alpha / \partial x$, etc.

We show that Cherkasov's condition is a special case of the results of $\S 3$; i.e., a much wider class of diffusion processes can be mapped one-to-one into the Wiener process than is indicated by the Cherkasov condition (4.2). First we reformulate Cherkasov's result in a more convenient way. In terms of Cherkasov's variables (4.1), one can show that $D\left(t^{\prime}, x^{\prime}\right)$ defined by (2.10) satisfies

$$
D\left(t^{\prime}, x^{\prime}\right)=\frac{\gamma(t, x)}{2 \alpha(t, x)} \text {. }
$$

Then Cherkasov's result reduces to $\{\alpha, D\}$ satisfying

$$
\alpha(t, x) \frac{\partial^{2} D}{\partial x^{2}}+\alpha_{x}(t, x) \frac{\partial D}{\partial x}=0 .
$$

But the operator $\partial / \partial x^{\prime}=\alpha(t, x) \partial / \partial x$, so that Cherkasov's result (4.2) becomes equivalent to

$$
\frac{\partial^{2} D\left(t^{\prime}, x^{\prime}\right)}{\partial\left(x^{\prime}\right)^{2}}=0
$$

or

$$
\frac{\gamma(t, x)}{2 \alpha(t, x)}=p_{0}(t)+p_{1}(t) \cdot \int_{x_{0}}^{x}[a(t, z)]^{-1 / 2} d z,
$$

where $\left\{p_{0}(t), p_{1}(t)\right\}$ are arbitrary functions of $t$.

In terms of $D\left(t^{\prime}, x^{\prime}\right)$, using (2.11), (2.14) and (3.1), Theorem 2 can be restated to say that a diffusion process can be mapped one-to-one into a Wiener process if and only 
if $D\left(t^{\prime}, x^{\prime}\right)$ satisfies the p.d.e.

$$
\frac{\partial^{2}}{\partial\left(x^{\prime}\right)^{2}}\left[\frac{\partial D}{\partial t^{\prime}}+D \frac{\partial D}{\partial x^{\prime}}+\frac{\partial^{2} D}{\partial\left(x^{\prime}\right)^{2}}\right]=0 .
$$

Note that Cherkasov's result, corresponding to $D\left(t^{\prime}, x^{\prime}\right)$ satisfying $\partial^{2} D / \partial\left(x^{\prime}\right)^{2}=0$, is a special solution class of (4.6). It should also be noted that if $n\left(t^{\prime}, x^{\prime}\right)$ satisfies the backward heat equation, then

$$
D=\frac{2 \partial n / \partial x^{\prime}}{n}
$$

solves (4.6), generating another special solution class of (4.6).

In terms of the original variables (4.6) is equivalent to $\{\alpha(t, x), b(t, x), D\}$ satisfying the p.d.e.

$$
\begin{aligned}
\alpha^{3} \frac{\partial^{4} D}{\partial x^{4}} & +\left[\alpha b+5 \alpha^{2} \alpha_{x}\right] \frac{\partial^{3} D}{\partial x^{3}} \\
& +\left[2 \alpha b_{x}+\alpha_{x} b+4 \alpha\left(\alpha_{x}\right)^{2}+2 \alpha^{2} \alpha_{x x}\right] \frac{\partial^{2} D}{\partial x^{2}} \\
& +\left[\alpha b_{x x}+\alpha_{x} b_{x}\right] \frac{\partial D}{\partial x}+\alpha_{x} \frac{\partial^{2} D}{\partial t \partial x}+\alpha \frac{\partial^{2} D}{\partial t(\partial x)^{2}}=0
\end{aligned}
$$

For the important special case where the coefficients of Kolmogorov's equation (2.1) are independent of $t$,

$$
D=\frac{2 b(x)-a^{\prime}(x)}{2[a(x)]^{1 / 2}}, \quad x^{\prime}=\int_{x_{0}}^{x}[a(z)]^{-1 / 2} d z .
$$

Here Cherkasov's result reduces to requiring

$$
D=p_{0}+p_{1} \int_{x_{0}}^{x}[a(z)]^{-1 / 2} d z,
$$

where $p_{0}$ and $p_{1}$ are arbitrary constants for some fixed $x_{0}$. Theorem 2 corresponds to the generalization $D=l^{\prime} / l$, where $l\left(x^{\prime}\right)$ satisfies the confluent hypergeometric type equation

$$
\frac{d^{2} l}{d\left(x^{\prime}\right)^{2}}+\left[r_{0}+r_{1} x^{\prime}+r_{2}\left(x^{\prime}\right)^{2}\right] l=0,
$$

where $\left\{r_{0}, r_{1}, r_{2}\right\}$ are arbitrary constants. Here Theorem 2 allows $D$ to have four arbitrary constants whereas Cherkasov's result allows two arbitrary constants. The special subcase where $a(x)=$ const., corresponds to $D=b(x)$ and $x^{\prime}=x$ in (4.9) and (4.10).

Acknowledgments. I express my sincere thanks to Professors Jack Cowan and Henry Tuckwell for their encouragement. I am most indebted to Sukeyuki Kumei for bringing Lie's work to my attention and for translating it.

\section{REFERENCES}

[1] G. W. Bluman And J. D. Cole, Similarity Methods for Differential Equations, Springer-Verlag, New York, 1974.

[2] - The general similarity solution of the heat equation, J. Math. Mech., 18 (1969), pp. 1025-1042. 
[3] I. D. Cherkasov, On the transformation of the diffusion process to a Wiener process, Theor. Probability Appl., 2 (1957), pp. 373-377.

[4] A. Kolmogoroff, Über die analytischen Methoden in der Wahrscheinlichkeitsrechnung, Math. Ann., 104 (1931), pp. 415-458.

[5] SOPHUS LIE, On the integration of a class of linear partial differential equations by definite integrals (in German), Arch. for Math., 6 (1881), pp. 328-368.

[6] LuIGI M. RiCCIARDI, On the transformation of diffusion processes into the Wiener process, J. Math. Anal. Appl., 54 (1976), pp. 185-199. 